\title{
Model scientists
}

Many people doubt that human activity is warming the Earth's climate. The scepticism sometimes starts from a reasonable concern - that our best predictions of future climate come from large-scale computational models of the Earth's atmosphere, oceans and biosphere, and we can't completely trust them. Given the extreme complexity of the Earth climate system, we just don't yet have anything like a firm grasp on sources of possible error.

Most scientists, I think, would agree, even if they certainly wouldn't leap to the conclusion, as many sceptics do, that we should therefore not worry about climate change, or that recent temperature fluctuations probably have a natural origin. Doubts about how much to trust complex models offer no grounds whatsoever for trusting naive intuition, based on vague and wholly inexplicit models, instead.

But that error aside, it is legitimate and important to ask about what we can and cannot trust in the output of today's best climate models, which are relied on by scientists to form their views of climate risks. Can we believe everything? Nothing? Or something in between? The answer, which is not at all surprising, is probably something in between, but certainly enough, it appears, to be pretty sure of a number of consequential facts.

For example, one thing climate scientists think they know with reasonable certainty is the total change in global average temperature likely to result from an additional quantity of $\mathrm{CO}_{2}$ in the atmosphere. In 2009, a well-known study by H. Damon Matthews and colleagues (Nature 459, 829-832; 2009) concluded that the expected temperature change per unit of carbon emitted is close to being independent of both the initial level of atmospheric $\mathrm{CO}_{2}$ and how fast new $\mathrm{CO}_{2}$ is added. You get about $1.0-2.1^{\circ} \mathrm{C}$ per trillion tonnes of carbon (TtC) emitted no matter how you do the emitting.

That's pretty remarkable, and it's a strong claim given the complexity of the system and the underlying physics. How can we be sure of this? Well, it turns out that simulations of climate models play a big role. Matthews et al. first ran simulations using the University of Victoria Earth System Climate Model, a model of intermediate complexity coupling the climate system with atmospheric carbon and its radiative effects. They studied the long-term effects of

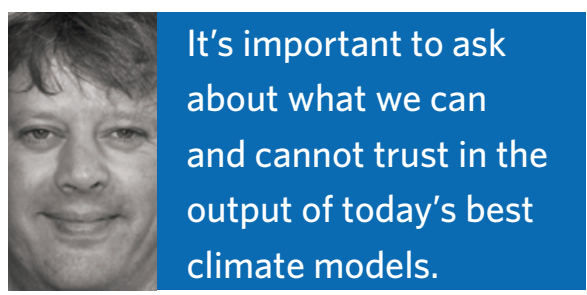

adding $\mathrm{CO}_{2}$ either gradually, $1 \%$ more per year, or suddenly, an increase of 2 or 4 times instantaneously, and found a similar result in all cases - about $1.7^{\circ} \mathrm{C}$ per TtC emitted.

Of course, no one would believe the results of just one model, but the authors compared their simulation results to those from 11 other climate models developed by other researchers around the globe, finding strong similarity. These groups all participated in the Coupled Carbon Cycle Climate Model Intercomparison Project (C4MIP) - an initiative to help foster the comparison of models and the explanation of differences between them. Most of these models, as well as the ensemble mean, showed a simple linear relationship between temperature change and cumulative emissions, and gave a value of temperature change per unit carbon emitted from 1.0 to $2.1^{\circ} \mathrm{C}$ per $\mathrm{TtC}$, with a mean of $1.6^{\circ} \mathrm{C}$ per $\mathrm{TtC}$.

Now, it is possible, of course, that all these different groups left some really decisive factors out of their models, or all made the same fatal error, which makes all the simulation results systematically inaccurate and misleading. But further development of the models since then has not changed these projections significantly. More or less identical results emerged from 15 different models compared during CMIP5, the most recent 5th phase of the CMIP project (see Nathan Gillett et al., J. Clim. 26, 6844-6858; 2013). And, as the original paper by Matthews et al. noted, these simulation estimates also agree with empirical estimates based on data over the past century.

This is how climate scientists have gained confidence in some basic insights, but only in some. What is known co-exists with a vast sea of uncertainty. How much will the global average temperature increase over the next, say, eight years? The models can't say, because their accuracy on decadal timescales is especially low - this falls into a no man's land between the timescales that the models handle well. Studies of the sources of prediction uncertainty - uncertainty in the models, inherent variability of climate itself, and uncertainty in the human response to climate change - suggest that models generate the highest signal-to-noise ratio, and should therefore be most useful, in the rough interval from 30 to 80 years ahead.

The models do give confidence that, for the most likely scenarios of human $\mathrm{CO}_{2}$ emissions over the next century, global and annual mean temperature will rise by around $2-4{ }^{\circ} \mathrm{C}$. They also strongly suggest, with a uniformity of results among models, that temperatures over land will generally rise about $50 \%$ more than over oceans, that the Earth's polar regions will warm more than elsewhere, and that the hydrological cycle will get stronger.

But which region will warm the most, where will it get colder, and who will get more or less rain? Again, lots of uncertainty. Model outputs for regional and decadal predictions vary strongly from one model to another, or even between different runs of the same model, so these features simply cannot currently be predicted. That's modelling. It gets better, but gradually, and always exists at a stage where some things can be known and others cannot.

In this sense, climate modelling doesn't really seem so different from any other area of science where it's really hard to do experiments on a system of interest weather, obviously, but also epidemiology or computational astrophysics. Making progress requires public competition between different models and ideas, and this is what climate scientists have been doing for the past four decades. Competition was set up originally in 1995 in the initial Coupled Model Intercomparison Project, which established a database for climate scientists of coupled global climate model simulations using standardized boundary conditions. Climate scientists since then have been able, independently, to examine the outputs of different simulations, and their physical content, to try to find out why different models give different outputs in response to the same input - or to see which kinds of outputs are common to all models and ought to be trusted, at least tentatively.

All of these models are works in progress, still crude in many areas, and capable of being made much better. And they will get better, through the ordinary, messy, cumulative process of science.

\section{MARK BUCHANAN}

